\title{
ESTIMACION DEL PODER DE MERCADO EN LA INDUSTRIA DEL COBRE DE ESTADOS UNIDOS
}

\author{
MEASURING MARKET POWER IN THE US COPPER INDUSTRY
}

\section{ANDRES LUENGO*}

Departamento de Administración

Pontificia Universidad Javeriana

\begin{abstract}
The study of the relationship between the structure of the industry and market power has been one of the most discussed topics in microeconomic literature. In this research, we focus on the "New Empirical Industrial Organization" (NEIO) approach and particularly on the models developed by Bresnahan (1982) and Lau (1982), and Porter (1983) to analyse the effect of several mergers and acquisitions that took place in the US copper Industry during the period 1994-2009 on market power. We found that although the industry was highly concentrated, the results suggest that mergers and acquisitions had no effect on market power and the industry remained competitive during this period.
\end{abstract}

Keywords: Market power, new empirical industrial organization, copper industry.

JEL Classification: D40, D43, L13, L61, L72.

\section{Resumen}

El estudio de la relación entre la estructura de la industria y el poder de mercado ha sido uno de los temas más discutidos en la literatura microeconómica. En esta investigación se adopta el enfoque de la "Nueva Organización

\footnotetext{
* Departamento de Administración de la Facultad de Ciencias Económicas y Administrativas de la Pontificia Universidad Javeriana. Cra.7 No. 40B-36, Piso 4, Edificio No. 20 Jorge Hoyos Vásquez, S.J. Bogotá, Colombia. E-mail: andres.luengo@javeriana.edu.co
} 
Industrial Empírica" (NOIE) y se siguen los modelos desarrollados por Bresnahan (1982) y Lau (1982), y Porter (1983) para analizar el efecto de varias fusiones y adquisiciones sobre el poder de mercado de la industria del cobre en Estados Unidos durante el período 1994-2009. Se destaca que, aunque el análisis de la industria muestra una mayor concentración, los resultados sugieren que las fusiones y adquisiciones no tuvieron efecto sobre el poder de mercado y que la industria mantuvo una conducta competitiva durante el período analizado.

Palabras clave: Poder de mercado, nueva organización industrial empírica, industria del cobre.

Clasificación JEL: D40, D43, L13, L61, L72.

\section{INTRODUCCION}

La estimación empírica de la conducta de las industrias ha sido ampliamente debatida en la literatura de la organización industrial. La principal discusión se ha centrado en la observabilidad de los costos marginales, lo que ha derivado en dos grandes enfoques de investigación: primero, el tradicional enfoque "Estructura-ConductaResultado" (ECR), que se ha caracterizado por el análisis del poder de mercado a partir de la relación entre las medidas de beneficio e indicadores de concentración de la industria. En segundo lugar, debido a la dificultad de observar directamente las medidas de beneficio o los costos marginales surge la Nueva Organización Industrial Empírica (NOIE), que se caracteriza por inferir la conducta de mercado a través de la respuesta de los precios a cambios en las elasticidades de la demanda y a variaciones en los componentes de los costos.

La estimación de la conducta competitiva en la industria de commodities ha sido ampliamente debatida en la literatura, por ejemplo, Suslow (1986) estimó un modelo estructural para medir el poder de mercado en la industria del aluminio en el período entre las dos guerras mundiales. Graddy (1995) estimó el poder de mercado y diferencias de precios pagadas por diferentes tipos de consumidores en el mercado de pescados al por mayor de Fulton. Por otra parte, Igami (2015) utiliza un modelo de oligopolio para evaluar el impacto del cartel internacional del café sobre los precios y el bienestar global.

Sin embargo, como la mayoría de los modelos empíricos, este enfoque no ha estado exento de críticas. En primer lugar, Corts (1999) muestra que niveles intermedios de conducta anticompetitiva medidos por el parámetro de conducta pueden subestimar el verdadero poder de mercado si este resultado no es un equilibrio de una variación conjetural. Como consecuencia, el método del parámetro de conducta solo podría ser utilizado para identificar si la industria presenta una conducta competitiva, monopolística o a la Cournot (Zeidan y Rosende, 2009). Algunos estudios empíricos 
que han validado la metodología del parámetro de conducta son, Genesove y Mullin (1998) y Wolfram (1999) para la industria del azúcar en Estados Unidos y el mercado spot de la electricidad en el Reino Unido, respectivamente.

Aunque reconocemos la importancia de estas críticas, en este artículo seguimos a Igami (2015) e interpretamos el parámetro de conducta como el grado promedio de colusión en la industria, lo que puede ser interpretado como un análisis de forma reducida de todas las posibles interacciones estratégicas de la industria, lo que nos permite distinguir entre los distintos equilibrios de precios observados en los datos. De acuerdo a esto, en esta investigación se considerará la propuesta teórica de la NOIE, y específicamente las contribuciones seminales de los modelos de oligopolio desarrollados por Bresnahan (1982) y Lau (1982) y Porter (1983), para estimar el poder de mercado en la industria del cobre en Estados Unidos y analizar si la ola de fusiones y adquisiciones que ha experimentado esta industria durante los últimos años ha dado lugar a algún efecto sobre la estructura competitiva del mercado en un contexto en el cual los costos marginales de la industria no están disponibles.

En particular, se considera la industria del cobre de Estados Unidos debido a que esta industria aparentemente presenta una alta concentración y se caracteriza por la presencia de grandes empresas especializadas en la extracción de recursos naturales. Guzmán (2007) plantea que la acción de las grandes empresas debería estar asociada a un comportamiento no competitivo y que la competencia perfecta no es una teoría realista para explicar la estructura competitiva de la industria global del cobre. Por su parte, Tilton et al. (1997) y Agostini (2006) señalan que la industria del cobre en Estados Unidos ha sido competitiva al menos desde 1980. Con todo esto, y dada la ola de fusiones y adquisiciones en esta industria, desde finales de los años noventa, y el boom del cobre a partir de la primera mitad del 2000, la industria del cobre de Estados Unidos se convierte en un atractivo objeto de estudio para la teoría de la organización industrial.

Metodológicamente, la propuesta de esta investigación plantea un modelo econométrico estructural biecuacional, demanda y relación de oferta, estimado por mínimos cuadrados en dos y tres etapas. Se utilizan datos mensuales para el período comprendido entre 1994 y 2009; los datos incluyen el consumo de cobre, precios del cobre y de sus substitutos, y medidas de ingreso por la parte de la demanda. Los datos que se consideran en la relación de oferta incluyen principalmente precios de los inputs y subproductos del cobre. En general, la información para esta industria es de difícil obtención dado que suele estar disponible solo para las empresas que componen la industria así como para algunas agencias internacionales que la compilan para investigación privada y confidencial. No obstante, la información sobre los precios de los minerales y del consumo del cobre fue obtenida gracias a la colaboración del International Copper Study Group (ICSG) y del U.S. Geological Survey (USGS) ${ }^{1}$.

1 El autor agradece a Daniel Elstein (especialista del cobre) del U.S. Geological Survey (USGS) y a Joseph C. Pickard (economista) del International Copper Study Group (ICSG) por facilitar la información estadística necesaria para la realización de esta investigación. 
El resto de las estadísticas fueron obtenidas por medio de varias fuentes y agencias gubernamentales de Estados Unidos, como el Bureau of Labor Statistics (BLS), el U.S. Energy Information Administration (EIA), el U.S Securities and Exchange Commission (SEC) y la Reserva Federal de Estados Unidos.

Los resultados de esta investigación sugieren que a pesar de la alta concentración de la industria, los precios han sido muy cercanos a los precios bajo competencia perfecta. Esto puede ser interpretado como que las empresas no han ejercido poder de mercado en el período analizado, por lo que la ola de fusiones podría no haber alterado la conducta competitiva de la industria.

Esta investigación se compone de cinco secciones incluyendo esta introducción. La sección dos presenta una caracterización de la industria del cobre, en la cual se describe su proceso productivo, así como la tendencia de los últimos años en la estructura de la industria. En la sección tres se presenta el planteamiento metodológico. En esta sección se definen las relaciones y ecuaciones que serán estimadas e interpretadas más adelante. La sección cuatro presenta los resultados empíricos de la estimación de los modelos estructurales y finalmente en la sección cinco se detallan las principales conclusiones.

\section{LA INDUSTRIA DEL COBRE}

El cobre refinado para uso industrial puede ser obtenido a través de dos procesos de acuerdo a las características propias de cada yacimiento: electroobtención (SX-EW) o flotación, representados en la Figura 1. Como se describe en Agostini (2006), estos procesos se pueden explicar en cuatro pasos: producción de mina, concentración, fundición y refinado. En el proceso de producción de mina se extrae la parte mineralizada y otros elementos del macizo rocoso de la mina por medio de los subprocesos de perforación y tronadura, carguío y transporte y el posterior chancado (trituración). El tratamiento del material chancado depende de si se trata de mineral oxidado (electroobtención) o mineral sulfurado (flotación), para el primero el material chancado es almacenado en una planta de lixiviación que separa los residuos y otros minerales del cobre para obtener un ánodo de cobre concentrado y purificado, mientras que para el segundo el material chancado es nuevamente molido y el cobre es separado de otros minerales e impurezas por medio de un proceso de flotación para obtener concentrado de cobre. Este último es posteriormente fundido para remover otros minerales como fierro o azufre, de lo cual se obtiene un ánodo de cobre 99,5\% puro. Finalmente, en la etapa de refinación los ánodos de cobre en forma de láminas son sumergidos en piscinas con una solución de agua y ácido sulfúrico y por medio de electrolisis se obtiene un cátodo de cobre con una pureza del 99,9\%, el cual es el producto final para el consumo industrial.

Hasta finales de los años setenta las empresas productoras de cobre primario se extendían hasta la industria de productos semifabricados. Sin embargo, la reestructuración 


\section{FIGURA 1}

EL PROCESO DE PRODUCCION DEL COBRE

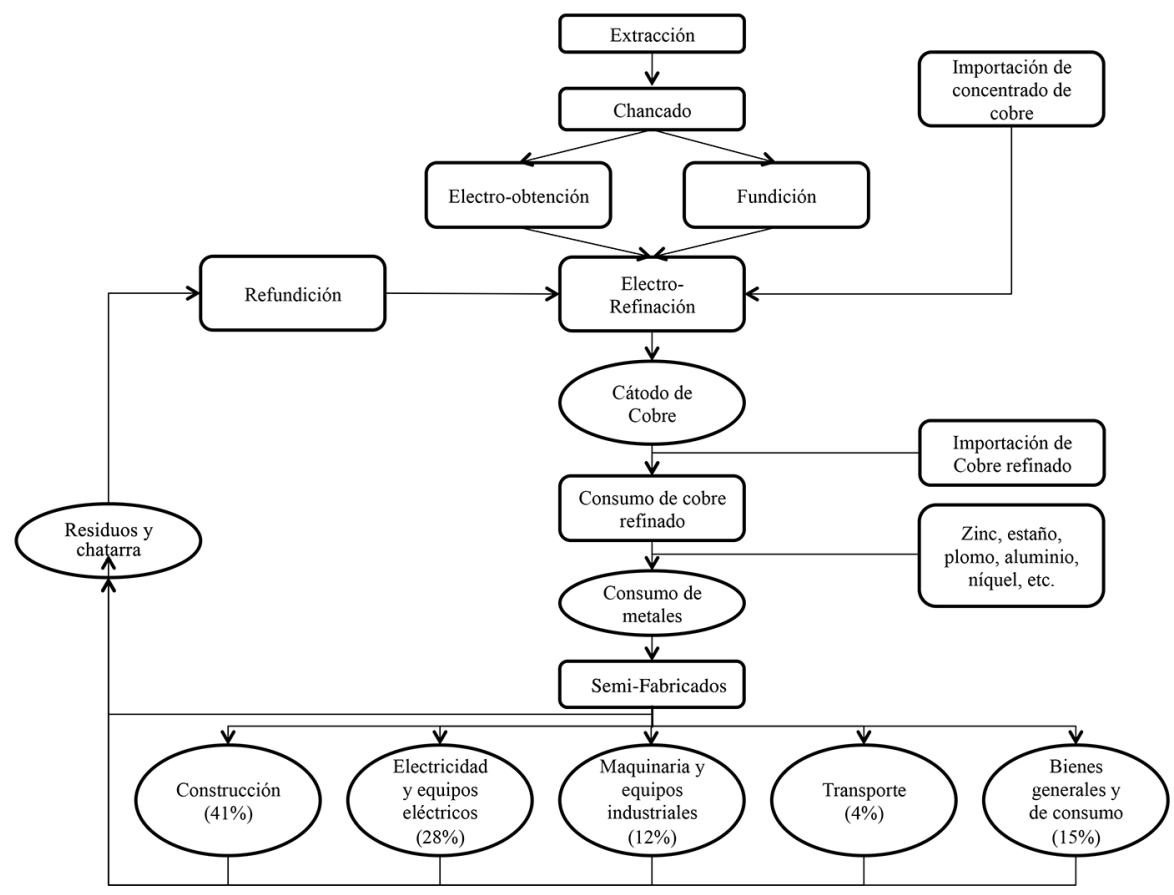

Fuente: Elaboración propia con base en CDA, Codelco.

de la industria de los años posteriores llevó a las empresas a especializarse en la producción. En cuanto al proceso productivo se destaca que este ha estado siempre verticalmente integrado debido principalmente a los altos costos de transporte del material rocoso que contiene aproximadamente solo un $1 \%$ en promedio de cobre, mientras el $99 \%$ restante son residuos y otros minerales (Agostini, 2006). Las fases de electroobtención y electrorrefinación requieren grandes cantidades de electricidad, que equivalen a alrededor del $12 \%$ del costo de producción de una libra de cobre refinado. Otros costos variables importantes en el proceso de producción son los costos laborales, 15\%; el petróleo, 7\%; el acero, 4\%; y otros insumos, 28\% (Cochilco, 2008). Los costos unitarios totales incluyen costos fijos tales como impuestos y seguros, los cuales se aplican ya sea que la empresa opere o no.

La producción de cobre de mina de Estados Unidos durante el período de estudio (1994-2009) presenta una tendencia a la baja, pasando de 1,81 millones de toneladas en 1994 a 1,31 millones de toneladas en 2008. Sin embargo, durante ese período 
Estados Unidos se mantuvo como segundo productor mundial con un $8,5 \%$ de la producción en $2008^{2}$, solo superado por Chile que concentra el $35 \%$ de la producción en el mismo año (USGS, 2008).

A pesar de mantener una posición estable a nivel internacional, la industria de cobre en Estados Unidos ha sufrido grandes transformaciones a nivel doméstico siguiendo la tendencia de reestructuración internacional de la industria, denominada la era de la súper consolidación (PricewaterhouseCoopers, 2007). En la primera columna del Cuadro 1 se puede observar el cambio de propiedad de las mayores minas productoras de cobre y de las cinco mayores empresas operadoras desde 1994 hasta el año 2008, año en el que las grandes empresas productoras mundiales han tomado el control de la producción doméstica en Estados Unidos. Una de las mayores y más citadas megacompras en la industria del cobre americano fue la de Phelps Dodge Corp. por parte de Freeport-McMoRan Copper \& Gold Inc. (FCX), por la suma de 26 billones de dólares (USGS, 2006), transformándose así en el principal productor de cobre en Estados Unidos y segundo a nivel mundial. Previo a esta adquisición, durante junio de 1999, Cyprus Amax y ASARCO Inc. anunciaron la fusión de ambas empresas, lo que las transformaría en el segundo productor mundial. Sin embargo, en agosto del mismo año ambas empresas anunciaron el rechazo al intento de compra hostil por parte de Phelps Dodge Corp. apelando a las leyes antimonopólicas del gobierno norteamericano. En septiembre de 1999 el Grupo México (GM) entra en una disputa con Sterlite Industries (India) por hacerse con el 100\% de la propiedad de ASARCO Inc., lo que tuvo como consecuencia la disolución de la fusión entre ASARCO Inc. y el Cyprus Amax Corp., las que finalmente fueron adquiridas en el tercer trimestre de 1999 por GM y Phelps Dodge Corp., respectivamente (USGS, 1999).

Por otra parte, la empresa Magma Copper Co. fue adquirida en enero de 1996 por la empresa australiana Broken Hill Propietary Co. Ltd. (BHP) en una transacción que significó 1,8 billones de dólares, transformando a BHP en el segundo mayor productor de cobre mundial en ese momento. En los años posteriores, BHP decide cerrar varios de sus yacimientos en Estados Unidos debido a los altos costos de explotación y a las bajas leyes de los yacimientos, por lo que la mayor parte de su producción se originó en sus yacimientos de Australia y Sudamérica.

Como consecuencia de lo anterior y producto de las desnacionalizaciones de los años noventa la industria del cobre ha tendido en forma creciente a concentrarse durante los últimos años (O’Ryan, 2002). Es así como los cinco mayores productores de Estados Unidos concentraron más del 97\% de la producción en 1994 y más del 90\% en 2008; y de la misma manera concentraban la propiedad de 13 minas en 1994 y 14 de las 18 principales minas productoras del ranking del USGS para 2008.

2 Estados Unidos perdió a manos de Perú el segundo lugar de la producción mundial en el año 2007 retomándolo en 2008. Sin embargo Perú se ha consolidado como el segundo productor mundial de cobre por detrás de Chile durante el año 2009, con una creciente alza en la producción y en las inversiones, por lo que se prevé que esta posición se mantendrá invariante por los próximos años. 


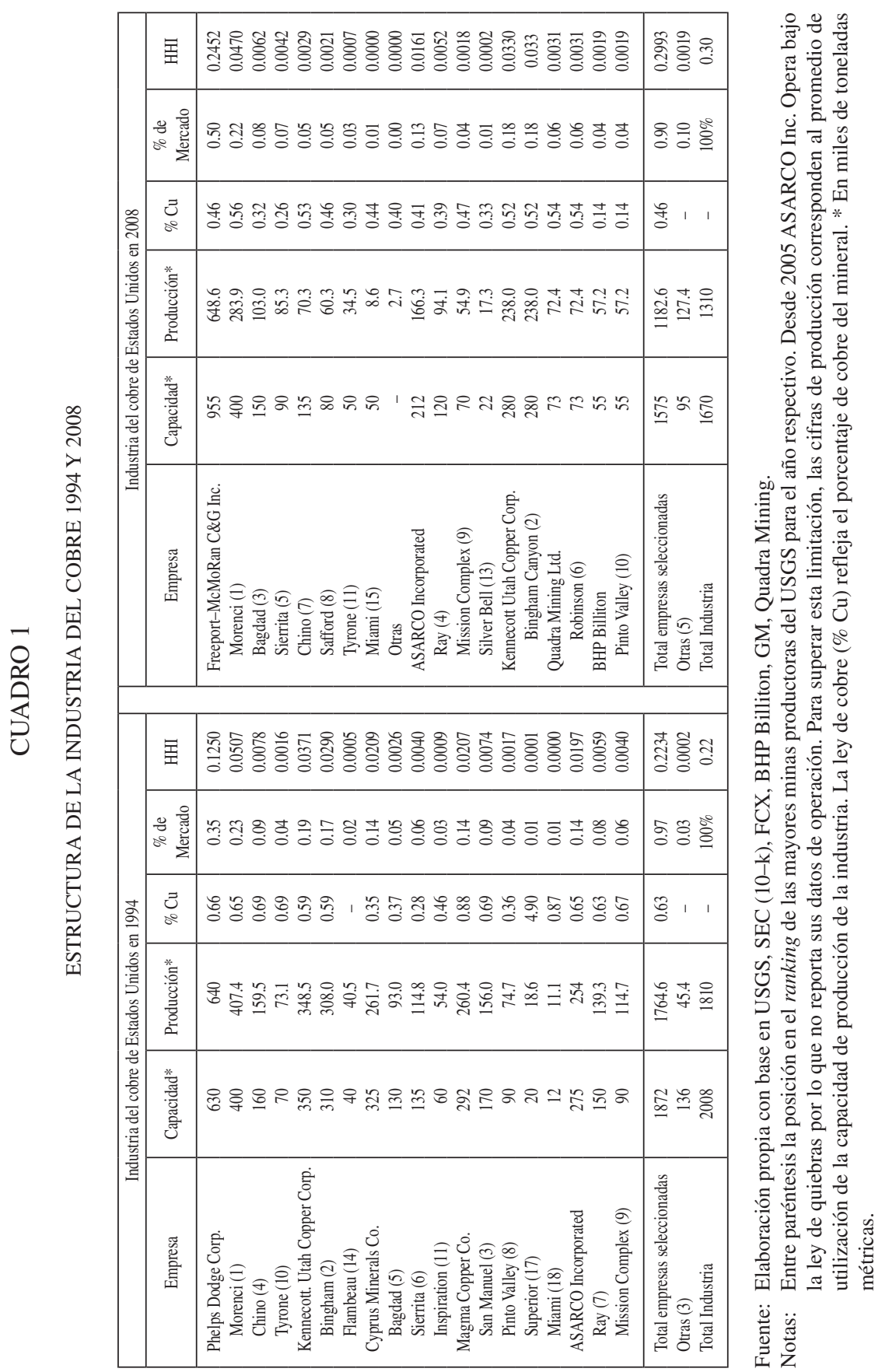


Al calcular el índice de Herfindahl-Hirschman (HHI) ${ }^{3}$ en el mercado del cobre en Estados Unidos para el año 1994 y 2008, se observa que es superior a 1.800 puntos, el límite fijado por las autoridades reguladoras de la competencia en Estados Unidos y a partir del cual el poder de mercado podría ser preocupante, este índice apoya la hipótesis de concentración de O'Ryan (2002), pasando de 22\% (2.200 puntos) en 1994 a 30\% (3.000 puntos) en 2008. Sin embargo, el poder de mercado no está determinado solo por la participación de mercado de las empresas sino también por la elasticidad precio de la demanda, la posibilidad de nuevos entrantes, las acciones estratégicas de las empresas y el comercio multilateral (Alvarado et al., 1998), por lo que considerar solo el índice de Herfindahl-Hirschman en el análisis podría conducir a conclusiones erróneas acerca del poder de mercado de la industria.

El análisis anterior muestra que la industria del cobre en Estados Unidos ha sido dominada por pocas empresas de gran tamaño. Guzmán (2007) plantea que la acción de las grandes empresas debería estar asociada con un comportamiento no competitivo y que la competencia perfecta no es una teoría realista para explicar la estructura competitiva global en esta industria. Sin embargo, Agostini (2006) y Tilton et al. (1997) señalan que la industria en Estados Unidos ha sido competitiva al menos desde el año 1980. Por lo tanto, es posible que estas grandes empresas también sean tomadoras de precios y que, como sugiere el mismo Guzmán (2007), han debido sortear las dificultades del mercado por medio de recortes de producción y además a través de fusiones y adquisiciones.

\section{MODELOS}

A partir de finales de los años setenta es posible identificar un importante cambio en la orientación del trabajo empírico de la economía industrial. Los economistas de este campo, insatisfechos con las hipótesis fundamentales del paradigma clásico ECR, desarrollaron un nuevo concepto denominado por Bresnahan (1989) como la "Nueva Organización Industrial Empírica" (NOIE). De acuerdo a este autor el enfoque de la NOIE está basado en modelos de competencia imperfecta, el supuesto de maximización de beneficios y en la estimación de la conducta de la industria a través de modelos econométricos estructurales de series de tiempo.

De acuerdo a Perloff et al. (2007), el nuevo enfoque tiene dos ventajas claves frente al paradigma clásico ECR: primero, en el nuevo enfoque los costos marginales y el poder de mercado pueden ser estimados simultáneamente usando modelos estructurales. Y segundo, es posible determinar cómo los costos u otros factores exógenos que determinan a estos modelos afectan a la conducta de la industria. Un estudio típico de

3 El índice de Herfindahl-Hirschman (HHI) es la herramienta mas utilizada para medir la concentración de mercado. Este índice se define como la sumatoria del cuadrado de las participaciones de mercado (expresadas como porcentaje) de cada una de las empresas en la industria (Perloff et al., 2007). 
la NOIE que intente identificar el parámetro de conducta en un mercado oligopólico, se basa en la respuesta de los precios a cambios en la elasticidad de la demanda y los componentes de los costos (Zeidan y Rosende, 2009).

Considerando la propuesta teórica de la NOIE, en esta investigación se seguirán las contribuciones seminales de los modelos de oligopolio desarrollados por Bresnahan (1982) y Lau (1982); y Porter (1983).

\subsection{Modelo de oligopolio de Bresnahan (1982) y Lau (1982)}

Un punto de partida de un modelo característico de la NOIE es suponer que una industria está compuesta por $n$ empresas que producen un bien homogéneo con la misma tecnología. En este modelo la función de demanda de mercado puede ser escrita como:

$$
Q=Q(P, Z)
$$

donde $P$ es el precio, $Q$ la cantidad de producción o consumo y $Z$ es un vector de variables exógenas tales como ingreso o precio de los substitutos que afectan la curva de demanda, pero no al costo marginal. La función inversa de demanda asociada queda como:

$$
P=P(Q, Z)
$$

La curva de costo marginal puede ser escrita como:

$$
M C=C(Q, W)
$$

donde $W$ es un vector de variables exógenas, tales como el precio de los factores de producción que afectan al costo marginal de la industria, pero no a la función de demanda.

El ingreso total se define como el producto del precio y la cantidad demandada, por lo que la función de ingreso marginal queda como:

$$
M R=P(Q, Z)+Q\left[\frac{\partial P(Q, Z)}{\partial Q}\right]
$$

De acuerdo a Bresnahan (1982) y Lau (1982), es posible utilizar un parámetro de conducta, $\lambda$, que represente la estructura del mercado. Por lo que la ecuación (4) puede ser reescrita como una función de ingreso marginal percibido, de la forma: 


$$
M R(\lambda)=P(Q, Z)+\lambda Q\left[\frac{\partial P(Q, Z)}{\partial Q}\right]
$$

donde $\partial P(Q, Z) / \partial Q$ es la pendiente de la curva de demanda. Por lo tanto, $\lambda$ es un nuevo parámetro que mide el poder de mercado: si $\lambda=0$, el ingreso marginal es igual al precio por lo que la industria es competitiva; si $\lambda=1$, la industria actúa como monopolio o cartel perfecto; mientras que valores intermedios de $\lambda$ corresponden a otros conceptos de solución de oligopolio, como por ejemplo el equilibrio de Cournot, donde $\lambda=1 / n$, cada empresa tiene la misma participación de mercado en condiciones de equilibrio.

La condición de equilibrio de la industria se logra cuando el ingreso marginal percibido, ecuación (5), es igual al costo marginal, ecuación (3), lo que adopta la forma:

$$
M R(\lambda)=P(Q, Z)+\lambda Q\left[\frac{\partial P(Q, Z)}{\partial Q}\right]=M C(Q, W)
$$

reescribiendo esta ecuación es posible obtener la denominada "relación de oferta" (Bresnahan, 1982), que engloba la posibilidad de una conducta "no tomadora de precios", es decir, mantener el precio sobre el costo marginal.

No obstante, Bresnahan (1982) y Lau (1982) plantean que en la práctica, si la forma funcional de la demanda está mal especificada, el modelo presenta un severo problema de identificación del parámetro $\lambda$. Para ilustrar este problema se utiliza una función de demanda lineal, similar a la planteada por Bresnahan (1982), de la forma:

$$
Q=\alpha_{0}+\alpha_{1} P+\alpha_{2} Z+\mu_{d}
$$

Y una función de costo marginal como:

$$
M C=\beta_{0}+\beta_{1} Q+\beta_{2} W+\mu_{c}
$$

donde $\mu_{d} \mathrm{y} \mu_{c}$ son los términos de error con media cero y varianza constante. Por lo tanto la relación de oferta que corresponde a las ecuaciones (7) y (8) queda como:

$$
P=-\frac{\lambda}{\alpha_{1}} Q+\beta_{0}+\beta_{1} Q+\beta_{2} W+\varepsilon
$$

$\mathrm{Al}$ observar el sistema generado por las ecuaciones (7) y (9), se aprecia que ambas ecuaciones están identificadas, debido a que por una parte la ecuación de demanda (7) incluye solo una variable endógena, $P$, y excluye una variable exógena, $W$. Y del mismo modo, la relación de oferta (9) incluye una variable endógena, $Q$, y excluye una 
variable exógena, $Z$. Sin embargo, el parámetro de conducta, $\lambda$, no está identificado, lo cual se puede explicar al reescribir la ecuación (9) como:

$$
P=\beta_{0}+\gamma Q+\beta_{2} W+\varepsilon
$$

donde

$$
\gamma=\beta_{1}-\frac{\lambda}{\alpha_{1}}
$$

La ecuación (10) está claramente identificada dado que incluye una variable endógena, $Q$, y excluye una variable exógena, $Z$. No obstante, aún no es posible determinar la conducta de la industria debido a que no es posible determinar si el costo marginal es igual al precio o al ingreso marginal. Al despejar $\lambda$ de la ecuación (11) se observa que su identificación requiere la estimación de los parámetros $\beta_{1}, \alpha_{1}$ y $\gamma$, pero las ecuaciones (7) y (10) solo proveen información de los parámetros $\alpha_{1}$ y $\gamma$, por lo que $\lambda$ no podría ser estimado.

Dada la limitación señalada anteriormente, Bresnahan (1982) propone una solución mediante la modificación de la función de demanda. La modificación permite que alguna variable exógena sea capaz no solo de desplazar la curva de demanda sino también de cambiar su pendiente. Teniendo esto en cuenta, la ecuación de demanda (7) puede ser reescrita como:

$$
Q=\alpha_{0}+\alpha_{1} P+\alpha_{2} Z+\alpha_{3} P Y+\alpha_{4} Y+\mu_{d}
$$

La que puede ser reescrita como:

$$
Q=\left(\alpha_{0}+\alpha_{2} Z+\alpha_{4} Y\right)+\left(\alpha_{1}+\alpha_{3} Y\right) P+\mu_{d}
$$

donde $Y$ es una nueva variable exógena de la demanda como por ejemplo el ingreso o el precio de los substitutos. La primera parte del lado derecho de la ecuación (12a) puede ser interpretada como un intercepto vertical, mientras que la segunda parte como la pendiente. De acuerdo a Bresnahan (1982), la clave de la solución al problema de identificación es que $Y$ interactúa con $P$ de tal forma que cambios en $Z$ e $Y$ generan desplazamientos y rotación de la curva de demanda.

La modificación de la curva de demanda altera la relación de oferta (debido al problema de optimización), quedando de la forma:

$$
P=\frac{\lambda}{\alpha_{1}+\alpha_{3} Y} Q+\beta_{0}+\beta_{1} Q+\beta_{2} W+\varepsilon
$$


En este caso es posible estimar $\lambda$. Al definir $Q^{*}=-Q /\left(\alpha_{1}+\alpha_{3} Y\right)$, la ecuación (13) puede reescribirse como

$$
P=\lambda Q^{*}+\beta_{0}+\beta_{1} Q+\beta_{2} W+\varepsilon
$$

Si se considera a los parámetros $\alpha_{1}$ y $\alpha_{3}$ como conocidos ( $\hat{\alpha}_{1}$ y $\hat{\alpha}_{3}$, al estimar primero la ecuación de demanda) es posible estimar $\lambda$ y $\beta_{1}$ en la ecuación (13). Por lo tanto, $\lambda$ puede ser estimado en la ecuación (14).

\subsection{El modelo de Porter}

El modelo de Porter (1983) fue desarrollado para estudiar la interacción estratégica de la industria de ferrocarriles utilizando datos de series temporales de precios y niveles de producción agregados entre 1880-1886, posteriormente fue utilizado por Agostini (2006) para estudiar la conducta colusiva de la industrial del cobre en Estados Unidos antes de 1978. La principal diferencia con el modelo BL radica en la especificación de la ecuación de demanda y en que el modelo de Porter supone a la conducta de la industria como conocida en un período determinado. A continuación se presenta brevemente el desarrollo del modelo de Porter (1983), siguiendo a Agostini (2006), en adelante modelo AP.

Se sigue una función de demanda $\log$-lineal como:

$$
\log Q_{t}=\omega_{0}+\omega_{1} \log P_{t}+\omega_{2} \log Z_{t}+\mu_{d t}
$$

donde $Q_{t}$ y $P_{t}$ son la cantidad total demandada y el precio en el período $t$, respectivamente. $Z_{t}$ es una variable exógena tal como en el modelo anterior y $\mu_{d t}$ es el término de error. El parámetro $\omega_{1}$ es la elasticidad precio de la demanda que se supone negativa.

Utilizando datos agregados de la industria, la función de costo total queda como:

$$
T C_{t}=C\left(Q_{t}, W_{t}, \Gamma_{t}, \gamma_{i}, \mu_{c t}\right)
$$

donde $W_{t}$ es el vector de precios de los factores pagado por las empresas en el período $t, \Gamma_{t}$ son otras variables que cambian los costos, $\gamma_{i}$ son los parámetros desconocidos de la función de costo total y $\mu_{c t}$ es el término de error. La definición del costo marginal queda entonces como:

$$
M C_{t}=C_{1}\left(Q_{t}, W_{t}, \Gamma_{t}, \gamma_{i}, \mu_{c t}\right)
$$

Dado que las empresas ofrecen un bien homogéneo, todas tendrán el mismo precio en equilibrio, por lo que bajo el supuesto de maximización de beneficios, el problema de optimización queda como: 


$$
\max _{p_{t}} \pi_{t}=P\left(Q_{t}, \omega_{i}, \mu_{d t}\right) Q_{t}-C\left(Q_{t}, W_{t}, \Gamma_{t}, \gamma_{i}, \mu_{c t}\right)
$$

La condición de primer orden para este problema está dado por:

$$
Q_{t}+\frac{\partial Q_{t}}{\partial P\left(Q_{t}, \omega_{i}, \mu_{d t}\right)} P\left(Q_{t}, \omega_{i}, \mu_{d t}\right)-\frac{\partial C\left(Q_{t}, W_{t}, \Gamma_{t}, \gamma_{i}, \mu_{c t}\right)}{\partial Q_{t}} \frac{\partial Q_{t}}{\partial P\left(Q_{t}, \omega_{i}, \mu_{d t}\right)}=0
$$

La cual puede ser reescrita como:

$$
P\left(Q_{t}, w_{i}, \mu_{d t}\right)\left(1+\frac{1}{\eta}\right)=M C_{t}
$$

donde $\eta$ es la elasticidad precio de la demanda (parámetro $\omega_{l}$ de la ecuación (15)) y $M C_{t}$ es el costo marginal de la industria en el período $t$. El lado izquierdo de la ecuación (20) es el ingreso marginal del monopolio, el cual podría ser igual al precio en un modelo perfectamente competitivo. Por lo tanto, la ecuación (20) describe la oferta de monopolio, la cual se convierte en una función de oferta en el caso de competencia perfecta $(\eta=-\infty)$. Las acciones de las empresas bajo diferentes supuestos de conducta pueden ser definidas como:

$$
P\left(Q_{t}, w_{i}, \mu_{d t}\right)\left(1+\frac{\theta}{\eta}\right)=M C_{t}
$$

donde $\theta$ es un parámetro que mide la conducta del mercado. Reescribiendo la función de demanda en forma inversa como $P_{t}=P\left(Q_{t}, \omega_{i}, \mu_{d t}\right)$ y tomando logaritmos de las ecuaciones (17) y (15), la ecuación (21) puede ser reescrita como:

$$
\log P_{t}=\gamma_{0}+\gamma_{1} \log W_{t}+\gamma_{2} \log \Gamma_{t}-\log \left(1+\frac{\theta}{\omega_{1}}\right)+\varepsilon
$$

Como en Agostini (2006), un cambio de conducta en la industria permitirá identificar el parámetro de interés $\theta$. Así, si se define una variable dummy, $I_{t}$, igual a uno cuando la industria se encuentra bajo cualquier régimen cooperativo e igual a cero cuando la industria es perfectamente competitiva, y utilizando $I_{t}$ en la ecuación (22), la relación de oferta puede ser escrita como:

$$
\log P_{t}=\gamma_{0}+\gamma_{1} \log W_{t}+\gamma_{2} \log \Gamma_{t}+\gamma_{3} I_{t}+\varepsilon
$$

Por lo tanto los precios deberían ser mayores bajo un régimen cooperativo que en competencia perfecta, $\gamma_{3}$ debería ser positivo pero menor o igual que $\log \left[1+\left(1 / \omega_{1}\right)\right]$. 
Por lo tanto si la secuencia $\left\{I_{1}, \ldots, I_{t}\right\}$ es conocida, entonces las ecuaciones (15) y (23) pueden ser estimadas consistentemente utilizando mínimos cuadrados en dos etapas (Porter, 1983; y Agostini, 2006).

\section{MODELACION EMPIRICA Y RESULTADOS DE LA ESTIMACION}

\subsection{Análisis preliminar de datos}

La mayor parte de los datos de la industria del cobre en Estados Unidos se encuentran disponibles solo en fuentes privadas, sin embargo, luego de un intenso proceso de escrutinio de las fuentes de datos públicas, fue posible obtener información de la industria a nivel agregado en el U.S. Geological Survey (USGS) y en el International Copper Study Group (ICSG). La información que compilan el USGS y el ICSG proviene principalmente de los reportes de las empresas, asociaciones de productores, artículos de prensa y agencias privadas, entre otras fuentes ${ }^{4}$. Otras variables utilizadas en la investigación tales como variables de ingreso, salarios y precio de otros commodities se obtuvieron a través de agencias gubernamentales como la Bureau of Labor Statistics (BLS), el U.S. Energy Information Administration (EIA) y la Reserva Federal de Estados Unidos. La Tabla 1, muestra un resumen estadístico de los datos utilizados.

Los datos sobre el precio LME del cobre y del aluminio, en centavos de dólar por libra, fueron suministrados por el USGS para el período comprendido entre 19702009; el precio de la plata, en dólares por onza, fue suministrado desde 1990 hasta 2009. La información sobre consumo y producción de cobre, medida en miles de toneladas, fue suministrada por el ICSG para el período 1994-2009. Por su parte, el precio del petróleo, en dólares por barril WTI, y el de la electricidad, en centavos por $\mathrm{kWh}$ del sector industrial, fueron obtenidos desde la EIA, a partir de enero de 1986 y enero de 1976, respectivamente ${ }^{5}$. Como medida de la actividad económica se utiliza el índice de producción industrial (G.17) y para el costo de capital se usa la tasa de crédito corporativo (G.19), las cuales son publicadas por la Reserva Federal de Estados Unidos. Finalmente, el salario es medido como el ingreso promedio en dólares por hora de los trabajadores del sector primario publicados por el BLS. Todas las series estadísticas son de periodicidad mensual; ninguna ha sido ajustada estacionalmente; y todos los precios fueron deflactados por el índice de precios al productor del sector de commodities (año base 2002) publicado por el BLS.

\footnotetext{
4 Amparado en la legislación norteamericana, el USGS recibe también directamente de las empresas productoras información financiera y de producción. No obstante, esta información no está disponible públicamente.

5 El precio de la electricidad del sector industrial no se encuentra disponible, con una periodicidad mensual entre 1984 y 1990.
} 


\section{TABLA 1}

RESUMEN ESTADISTICO

\begin{tabular}{|l|l|l|r|r|r|r|}
\hline Variable & \multicolumn{1}{|c|}{ Nombre Variable } & Fuente & \multicolumn{1}{c|}{ Promedio } & \multicolumn{1}{c|}{ Dev. Est. } & \multicolumn{1}{c|}{ Max. } & \multicolumn{1}{c|}{ Min. } \\
\hline Q & Consumo de cobre en miles de toneladas & ICSG & 205.2895 & 34.3340 & 275.1324 & 115.0000 \\
Q1 & Consumo de cobre en millones de libras & ICSG & 452.5782 & 75.6922 & 606.5528 & 253.5273 \\
Z & Precio del aluminio LME & USGS & 65.1467 & 7.7319 & 85.7939 & 40.4483 \\
ZUS & Precio del aluminio US Market & USGS & 68.6077 & 7.8530 & 91.5164 & 42.1616 \\
P & Precio del cobre LME & USGS & 113.4129 & 46.4566 & 237.6767 & 63.2718 \\
PP & Precio del cobre productor & USGS & 117.9763 & 46.4756 & 248.6279 & 66.9757 \\
PRO & Producción de cobre & ICSG & 123.0697 & 27.8978 & 176.8502 & 83.4970 \\
W & Salarios de la industria del cobre & BLS & 14.6646 & 1.9373 & 18.0286 & 10.0979 \\
E & Precios de la electricidad & EIA & 4.3952 & 0.4327 & 5.5652 & 3.4899 \\
CPI & Índice de producción de la construcción & RF & 90.6596 & 8.6096 & 107.9249 & 70.2399 \\
OIL & Precio del petróleo & EIA & 29.8191 & 12.7637 & 68.2966 & 11.2829 \\
K & Costo de capital (Moody's AAA) & RF & 6.4733 & 0.9663 & 8.6800 & 4.9600 \\
IPI & Índice de producción industrial & BLS & 98.2139 & 8.1262 & 111.4722 & 79.2525 \\
IPE & Índice de precios de la electricidad & BLS & 33.1752 & 3.6570 & 43.5578 & 21.4852 \\
IOP & Índice de precios del petróleo & BLS & 146.4043 & 85.6289 & 464.0269 & 51.1225 \\
IAP & Índice de precios del aluminio & BLS & 126.5362 & 29.5155 & 201.8178 & 79.9814 \\
IPLAS & Índice de precios del Plástico & BLS & 125.8898 & 35.4125 & 205.4824 & 85.5959 \\
\hline
\end{tabular}

Aunque la mayoría de los datos están disponibles para un amplio margen temporal, el período de estudio de esta investigación se limita al comprendido entre 1994 y 2009. Esto último debido a que las variables de consumo y producción, que son utilizadas como variables dependientes en la ecuación de demanda, solo se encuentran disponibles para este período. Sin embargo, como plantea O’Ryan (2002), el período de fusiones y adquisiciones de la industria, idea central de esta investigación, se intensifica desde finales de los años noventa, con lo cual se justifica la validez teórica del período utilizado.

\subsection{Estimación empírica}

En cuanto a los procedimientos econométricos, se utilizaron mínimos cuadrados en dos etapas (MC2E) y tres etapas (MC3E) para estimar las ecuaciones de demanda y las relaciones de oferta, esta metodología permite asegurar la validez de los estadísticos usuales $\left(t, F, R^{2}\right)$. Para corregir los potenciales problemas de autocorrelación se utilizó un proceso $\mathrm{AR}(\mathrm{p})$; y con el fin de evitar cualquier error en la medición del parámetro de poder de mercado se siguió, tanto para la ecuación de demanda como la relación de oferta, una especificación general basada en los modelos estructurales.

\subsubsection{Estimación de la demanda}

La especificación de la demanda se basa en la teoría tradicional de la demanda, donde la cantidad demandada es afectada por su propio precio y por variables tales como 
medidas de ingreso y precios de los substitutos, que pueden desplazarla o modificar su pendiente tal como se presentó en la sección 3. Adicionalmente, la demanda del cobre se considera como una demanda derivada y sin ninguna identificación del productor, dado que el cobre es un producto homogéneo, que es utilizado principalmente en la fabricación de bienes intermedios que posteriormente son usados como insumos en la fabricación de bienes finales.

Considerando estos antecedentes, se especificó una función de demanda para el cobre como una función de su propio precio, el precio de un bien substituto y un indicador de la actividad económica. De acuerdo a García y Leuthold (1997), cualquier modelación de la función de demanda de un commodity debiera incluir estas tres variables. Por lo tanto, con base en la discusión anterior y siguiendo las ecuaciones (12) y (15) la función de demanda del modelo BL puede ser escrita como:

$$
\log Q_{t}=\alpha_{0}+\alpha_{1} \log P_{t}+\alpha_{2}\left[\log P_{t} \times \log Z_{t}\right]+\alpha_{3} \log Z_{t}+\alpha_{4} \log I P_{t}+\mu_{B L t}
$$

Mientras que para el modelo AP, la función de demanda puede ser especificada como:

$$
\log Q_{t}=\omega_{0}+\omega_{1} \log P_{t}+\omega_{2} \log Z_{t}+\omega_{3} \log I P_{t}+\mu_{A P t}
$$

donde $Q_{t}$ es el consumo de cobre, $P_{t}$ es el precio del cobre, $Z_{t}$ es el precio del aluminio, $I P_{t}$ es el índice de producción industrial y $\left[P_{t} Z_{t}\right]$ es el término de interacción de la función de demanda en el modelo BL.

Los resultados de la estimación de ambas ecuaciones de demanda se presentan en la Tabla 2. Se debe destacar que dado que uno de los parámetros claves en la estimación del poder de mercado en una industria es la elasticidad precio de la demanda, una estimación sesgada o inconsistente de sus coeficientes conducirá a conclusiones erróneas sobre la conducta de la industria (Agostini, 2006). Para resolver el posible problema de simultaneidad entre el precio y la cantidad demandada se utilizan como instrumentos del precio del cobre las variables de salario y el precio del petróleo.

En el modelo AP la elasticidad precio es el coeficiente estimado del precio en la ecuación de demanda. Sin embargo, en el caso del modelo BL la elasticidad precio y la elasticidad precio cruzada deben ser calculadas por sus primeras derivadas ${ }^{6}$. Las elasticidades estimadas se presentan en la Tabla 3.1.

La Tabla 3.2 muestra un resumen de la elasticidad precio estimada en distintos estudios aplicados a la industria del cobre. Comparando las tablas 3.1 y 3.2 , se observa que las estimaciones de la elasticidad precio se encuentran dentro del rango estimado

6 A partir de las estimaciones del modelo BL, se debe cumplir que: (1) La elasticidad precio de la demanda $\partial \log Q_{t} / \partial \log P_{t}=\alpha_{1}+\alpha_{2} \log Z_{t}<0$; y la elasticidad precio cruzada $\partial \log Q_{t} / \partial \log Z_{t}=\alpha_{3}+\alpha_{2} \log P_{t}>0$. 


\section{TABLA 2}

\section{DEMANDA}

\begin{tabular}{|c|c|c|c|c|c|c|}
\hline \multirow{3}{*}{ Variable } & \multicolumn{3}{|c|}{ Bresnahan \& Lau (1982) } & \multicolumn{3}{|c|}{ Agostini (2006) \& Porter (1983) } \\
\hline & & Modelo 1 & Modelo 2 & & Modelo 1 & Modelo 2 \\
\hline & $\mathrm{MCO}$ & (MC2E) & (MC3E) & $\mathrm{MCO}$ & (MC2E) & (MC3E) \\
\hline Constante & $74,2762^{*}$ & $25,5925^{*}$ & $28,1533^{*}$ & 104,3437 & 2,9050 **** & $2,8648^{* * *}$ \\
\hline Precio del cobre $\left(\mathrm{P}_{\mathrm{t}}\right)$ & $\begin{array}{r}0,041333 \\
(0,2956)\end{array}$ & $\begin{array}{r}-5,0675^{*} \\
(3,5681)\end{array}$ & $\begin{array}{r}-5,5912^{*} \\
(3,6665)\end{array}$ & $\begin{array}{r}-0,1831 * * * \\
(0,0467)\end{array}$ & $\begin{array}{r}-0,3836 * * * * \\
(0,0561)\end{array}$ & $\begin{array}{r}-0,3826 * * * \\
(0,0510)\end{array}$ \\
\hline Interacción de demanda $\left(\mathrm{P}_{\mathrm{t}} \mathrm{Z}_{\mathrm{t}}\right)$ & $\begin{array}{r}-0,00331 \\
(0,0043)\end{array}$ & $\begin{array}{l}1,1196^{*} \\
(0,8543)\end{array}$ & $\begin{array}{l}1,2443^{*} \\
(0,8780)\end{array}$ & & & \\
\hline $\begin{array}{l}\text { Índice de producción de la } \\
\text { construcción (CPI.) }\end{array}$ & $1,6716^{*}$ & $0,3143 * *$ & $0,3161 * *$ & $1,6289 * * *$ & $0,3845^{* *}$ & $0,3758^{* * *}$ \\
\hline & $(0,1934)$ & $(0,1863)$ & $(0,1866)$ & $(0,1850)$ & $(0,1750)$ & $(0,1594)$ \\
\hline Precio del aluminio $\left(\mathrm{Z}_{\mathrm{t}}\right)$ & $\begin{array}{r}0,6522 \\
(0,5722)\end{array}$ & $\begin{array}{l}-4,7632 \\
(4,0801)\end{array}$ & $\begin{array}{l}-5,3757 \\
(4,1945)\end{array}$ & $\begin{array}{r}0,2545 \\
(0,2445)\end{array}$ & $\begin{array}{r}0,5882 * * * * \\
(0,1373)\end{array}$ & $\begin{array}{r}0,6062^{* * * *} \\
(0,1238)\end{array}$ \\
\hline $\mathrm{AR}(1)$ & & $\begin{array}{r}0,6702 * * * \\
(0,0618)\end{array}$ & $\begin{array}{r}0,6844 * * * \\
(0,0599)\end{array}$ & & $\begin{array}{r}0,6392 * * * \\
(0,0635)\end{array}$ & $\begin{array}{r}0,6358^{* * * *} \\
(0,0563)\end{array}$ \\
\hline R2 Ajustado & 0,7261 & 0,7164 & 0,7107 & 0,7253 & 0,7492 & 0,7492 \\
\hline F-estadístico & 98,63 & 96,99 & 94,33 & 123,41 & 141,39 & 141,42 \\
\hline DW & & 2,1896 & 2,2007 & & 2,2181 & 2,2133 \\
\hline $\mathrm{N}^{\circ} \mathrm{Obs}$ & 192 & 191 & 191 & 192 & 189 & 189 \\
\hline
\end{tabular}

Notas: Error estándar en paréntesis. Cálculos propios utilizando el paquete econométrico EViews 5. * Significativo a un nivel del 10\%, ** Significativo a un nivel del 5\% y *** Significativo a un nivel del 1\% (para el modelo BL el contraste se hace a una cola y para el modelo AP se contrasta a dos colas).

TABLA 3.1

ELASTICIDADES DE LA DEMANDA

\begin{tabular}{|l|c|c|c|c|c|}
\hline & \multicolumn{2}{|c|}{ Bresnahan \& Lau (1982) } & & \multicolumn{2}{c|}{ Agostini (2006) \& Porter (1983) } \\
\cline { 2 - 3 } \cline { 5 - 5 } \cline { 5 - 5 } & Modelo 1 & Modelo 2 & Modelo1 & Modelo 2 \\
\hline Elasticidad precio (P) & $-0,40$ & $-0,40$ & & $-0,38$ & $-0,39$ \\
Elasticidad del IP (CPI) & 0,31 & 0,32 & 0,38 & 0,37 \\
Elasticidad cruzada (Z) & 0,45 & 0,42 & & 0,59 & 0,61 \\
\hline
\end{tabular}

Fuente: Elaboración propia con base en la tabla 2. Cálculos propios utilizando el paquete econométrico EViews 5. 
TABLA 3.2

ELASTICIDADES DE DEMANDA

\begin{tabular}{|l|c|c|c|}
\hline \multicolumn{1}{|c|}{ Autor } & Frecuencia & Periodo & Elasticidad \\
\hline Bozdogan and Hartman (1979) & anual & $1950-1973$ & $-0,47$ \\
Charles River Associates (1970) & anual & $1950-1967$ & $-0,21$ \\
Fisher, Cootner and Baily (1972) & anual & $1950-1966$ & $-0,21$ \\
Labys (1989) & anual & $1971-1985$ & $-0,39$ \\
Mackinnon and Olewiler (1986) & trimestral & $1947-1974$ & $-0,42$ \\
McNicol (1975) & anual & $1949-1966$ & $-0,33$ \\
Taylor (1979) & trimestral & $1956-1975$ & $-0,20$ \\
Thurman (1988) & mensual & $1975-1984$ & $-0,27$ \\
Vial (1988) & anual & $1965-1984$ & $-0,19$ \\
Wagenhals (1984) & anual & $1950-1980$ & $-0,35$ \\
Agostini (2006)* & mensual & $1950-1995$ & $-0,39$ \\
En esta investigación* & mensual & $1994-2009$ & $-0,39$ \\
\hline
\end{tabular}

Notas: (*) Corresponde a la elasticidad promedio de los modelos estimados.

Fuente: Adaptado de Agostini (2006), p. 29.

por otros autores, con coeficientes negativos y significativos, y ubicándose en el rango entre $-0,38$ y $-0,40$.

Como sugieren los analistas de mercado, se considera al aluminio como substituto del cobre; los resultados muestran que su elasticidad fue positiva y significativa en todos los modelos, con excepción del modelo BL1. Agostini (2006) presenta un resultado similar y plantea que este resultado puede ser explicado porque el precio del aluminio no es un precio de equilibrio, debido a que está sujeto a descuentos y recortes de producción por parte de los productores. Ante esta situación se utilizó el precio del plástico como substituto, pero dado que los resultados no fueron significativos en ninguno de los modelos, se optó por seguir utilizando al aluminio como substituto.

Por otra parte, el coeficiente del índice de producción industrial fue positivo, como se esperaba, pero no fue significativo, por lo que se optó por utilizar como medida de producción industrial el índice de producción industrial de la construcción, que resultó positivo y significativo en todos los modelos, con un coeficiente que varía entre 0,31 y 0,38 . Además, se consideró como medida de producción industrial el índice de producción industrial del sector de plásticos pero el modelo presentó una menor significancia de sus coeficientes y un menor $R^{2}$. Respecto al término de interacción $\left[P_{t} Z_{t}\right]$ en el modelo BL, no es posible interpretar su signo de manera directa, por lo que es considerado solo para el cálculo de las elasticidades.

Finalmente se especificaron las ecuaciones de demanda utilizando variables dummy para controlar los efectos de la crisis asiática y subprime, pero sus resultados no fueron significativos, por lo que se optó por omitirlas de la especificación. El mismo caso se presentó con la tendencia temporal. De acuerdo a los estadísticos Durbin-Watson (DW) obtenidos en las primeras estimaciones, se corrigió el problema 
de autocorrelación agregando un proceso AR(1), logrando coeficientes significativos y menores a uno en todos los modelos.

\subsubsection{Estimación de la oferta}

La evidencia empírica en la literatura económica sugiere una hipótesis alternativa a la visión tradicional de curvas de costo marginal y costo medio en forma de "U", mediante una curva de costos marginales constantes en algunas industrias (Foley y Clark, 1981). Así, siguiendo a Foley y Clark (1981), Guzmán (2007) y Agostini (2006), que plantean que un costo marginal constante es la mejor aproximación a los costos de la industria del cobre, se asume una función de producción con retornos constantes de escala, que permite especificar una función de costo marginal log-lineal constante.

Considerando la condición de equilibrio en (6) y utilizando (13), la relación de oferta para el modelo BL queda como:

$$
\log P_{t}=\beta_{0}-\left[\frac{\lambda}{\alpha_{1}+\alpha_{2} \log Z_{t}}\right] Q_{t}+\beta_{1} \log Q_{t}+\beta_{2} \log W_{t}+\beta_{3} \log O I L_{t}+\beta_{4} \log E_{t}+\beta_{5} \log S I L+\varepsilon_{B L t}
$$

De la misma forma para el modelo AP, considerando la condición de primer orden (21) y utilizando la ecuación (23), la relación de oferta puede ser escrita como:

$$
\log P_{t}=\gamma_{0}+\gamma_{1} \log W_{t}+\gamma_{2} \log O_{I} L_{t}+\gamma_{3} \log E_{t}+\gamma_{4} \log S I L+\gamma_{5} t+\gamma_{6} I_{t}+\varepsilon_{A t P}
$$

donde $W_{t}$ es el salario, $O I L_{t}$ es el precio del petróleo, $E_{t}$ es el precio de la electricidad, $S I L_{t}$ es el precio de la plata y $\lambda$ es el parámetro de conducta en el modelo BL, $t$ es la tendencia temporal e $I_{t}$ es la variable dummy que indica la existencia del régimen cooperativo de la industria en el modelo AP.

Los resultados de la estimación de ambas relaciones de oferta se presentan en la Tabla 4. Como en la ecuación de demanda, se corrige la autocorrelación por medio de un proceso AR(1) para el modelo AP y un proceso AR(2) para el modelo BL. En ambos casos los coeficientes estimados fueron significativos y los estadísticos DW derivados permanecieron en el rango entre 1,97 y 1,98 , muy cercanos al coeficiente 2 como sugiere la teoría.

Como se observa en la Tabla 4, en general, los coeficientes estimados presentaron los signos esperados y fueron en todos los modelos significativos. Sin embargo, desafortunadamente en las dos relaciones de oferta del modelo AP se obtuvo, contrario a lo esperado, un signo negativo en el salario. Una explicación puede ser que el salario de los trabajadores del sector primario ha permanecido vinculado a la producción y contexto económico más que a las variaciones de precios. No obstante, debido a su alto poder explicativo se optó por mantenerlo en la especificación. 


\section{TABLA 4}

OFERTA

\begin{tabular}{|c|c|c|c|c|}
\hline & \multicolumn{2}{|c|}{ Bresnahan \& Lau (1982) } & \multicolumn{2}{|c|}{ Agostini (2006) \& Porter (1983) } \\
\hline & Modelo 1 & Modelo 2 & Modelo1 & Modelo 2 \\
\hline & $(\mathrm{MC} 2 \mathrm{E})$ & (MC3E) & (MC2E) & (MC3E) \\
\hline Constante & $\begin{array}{c}0,7837 \\
(1,0547)\end{array}$ & $\begin{array}{c}0,8017 \\
(1,0141)\end{array}$ & $\begin{array}{c}4,6686 * * * \\
(0,9495)\end{array}$ & $\begin{array}{c}4,7204 * * * \\
(0,9187)\end{array}$ \\
\hline Cantidad (Q) & $\begin{array}{c}0,1434 * * * \\
(0,0589)\end{array}$ & $\begin{array}{c}0,1407 * * * \\
(0,0574)\end{array}$ & & \\
\hline Salario (W) & $\begin{array}{c}0,9053 * * * \\
(0,3035)\end{array}$ & $\begin{array}{c}0,8980 * * * \\
(0,2976)\end{array}$ & $\begin{array}{c}-0,9852 * * * \\
(0,2777)\end{array}$ & $\begin{array}{c}-1,0097 * * * \\
(0,2685)\end{array}$ \\
\hline Precio del petróleo (OIL) & $\begin{array}{c}0,1639 * * * \\
(0,0542)\end{array}$ & $\begin{array}{c}0,1641 * * * \\
(0,0530)\end{array}$ & $\begin{array}{c}0,2485 * * * \\
(0,0681)\end{array}$ & $\begin{array}{c}0,2550 * * * \\
(0,0662)\end{array}$ \\
\hline Precio de la plata (SIL) & $\begin{array}{c}0,1834 * * * \\
(0,0705)\end{array}$ & $\begin{array}{c}0,1840 * * * \\
(0,0689)\end{array}$ & $\begin{array}{c}0,9970 * * * \\
(0,2212)\end{array}$ & $\begin{array}{c}1,0101 * * * \\
(0,2126)\end{array}$ \\
\hline Poder de mercado $(\lambda)$ & $\begin{array}{c}0,0028 * * * \\
(0,0011)\end{array}$ & $\begin{array}{c}0,0028 * * * * \\
(0,0011)\end{array}$ & & \\
\hline Tiempo (t) & & & $\begin{array}{c}-0,0029 * * * \\
(0,0010)\end{array}$ & $\begin{array}{c}-0,0031 * * * \\
(0,0010)\end{array}$ \\
\hline I & & & $\begin{array}{c}0,1479 * * * \\
(0,0506)\end{array}$ & $\begin{array}{c}0,1499 * * * \\
(0,0489)\end{array}$ \\
\hline q & & & 0,0527 & 0,0532 \\
\hline $\mathrm{AR}(1)$ & $\begin{array}{c}1,2166 * * * \\
(0,0777)\end{array}$ & $\begin{array}{c}1,2196 * * * \\
(0,0760)\end{array}$ & $\begin{array}{c}0,8148 * * * \\
(0,0440)\end{array}$ & $\begin{array}{c}0,8079 * * * \\
(0,0427)\end{array}$ \\
\hline $\operatorname{AR}(2)$ & $\begin{array}{c}-0,2250 * * * \\
(0,0782)\end{array}$ & $\begin{array}{c}-0,2287 * * * \\
(0,0765)\end{array}$ & & \\
\hline R2 Ajustado & 0,9784 & 0,9784 & 0,9594 & 0,9599 \\
\hline DW & 1,9705 & 1,9746 & 1,9766 & 1,9793 \\
\hline $\mathrm{N}^{\circ} \mathrm{Obs}$ & 184 & 184 & 190 & 190 \\
\hline
\end{tabular}

Notas: Error estándar en paréntesis. Cálculos propios utilizando el paquete econométrico EViews 5. * Significativo a un nivel del 10\%, ** Significativo a un nivel del 5\% y *** Significativo a un nivel del 1\% (Para el modelo BL el contraste se hace a una cola y para el modelo AP se contrasta a dos colas).

El coeficiente del precio de la electricidad fue positivo, como se esperaba, pero no fue significativo, por lo que se optó por eliminarlo en ambos modelos. Por su parte, el coeficiente del precio del petróleo fue positivo y significativo en ambos modelos. En general, los resultados reflejan la importancia de los inputs en la extracción del cobre de mina; como se señala en COCHILCO (2008) estos inputs equivalen al 12\% y $7 \%$, respectivamente, del costo total.

Respecto al precio de la plata, su efecto es mayor en el modelo AP que en el modelo BL. En general, los estudios no consideran el uso de subproductos en la 
estimación de oferta, pero la significancia de su elasticidad confirma la importancia planteada por Agostini (2006) de incluir los subproductos del cobre en la estimación.

Considerando que ambos métodos (BL y AP) buscan estimar el poder de mercado utilizando diferentes metodologías, su análisis debe ser hecho de forma separada. En primer lugar, se sigue la estimación empírica tradicional del modelo BL y por razones de conveniencia la significancia de los parámetros es contrastada utilizando el test de una cola ${ }^{7}$. En este caso, es posible inferir directamente el poder de mercado de la industria analizando el parámetro $\lambda$, el cual es muy cercano a cero $(0,003)$, tanto en $\mathrm{MC} 2 \mathrm{E}$ como en MC3E. Estos resultados indican que la industria del cobre ha sido competitiva durante el período estudiado.

Ahora bien, dado que el valor es muy cercano a cero se realizan cuatro pruebas adicionales que confirmen los resultados. Primero, se prueba la estructura de monopolio, especificando $\lambda=1$. Segundo, se prueba un comportamiento a la Cournot con cinco empresas $(\lambda=1 / n)$, donde la hipótesis nula es $\lambda=0.2$ contra la hipótesis alternativa $\lambda \neq 0.2$. Tercero, se prueba la hipótesis de competencia perfecta fijando la hipótesis nula $\mathrm{H}_{0}: \lambda=0$. Cuarto, se calcula el índice de Lerner:

$$
L=\frac{P-M C}{P}=-\frac{\lambda Q_{t}\left(\partial P(\cdot) / \partial Q_{t}\right)}{P}=\frac{\lambda}{\eta}
$$

donde $\lambda$ es el poder de mercado y $\eta$ es la elasticidad precio de la demanda. El índice de Lerner por definición será cercano a cero bajo competencia perfecta $(\lambda=0)$. Por lo tanto, utilizando el poder de mercado y la elasticidad de la demanda se puede comprobar la conducta de la industria. En la Tabla 5.1 se presentan los resultados de las pruebas para ambas estimaciones del modelo BL (MC2E y MC3E).

Los resultados confirman que la conducta de la industria fue competitiva entre 1994 y 2009. No obstante, a pesar de rechazar al 1\% de significancia la hipótesis de competencia perfecta no indica que la estructura de la industria se aleje de una competitiva.

Por último, desde el punto de vista del análisis del poder de mercado en el modelo AP, como se mencionó en la sección 3, la ecuación (27) puede ser estimada consistentemente si la secuencia $\left[I_{1}, \ldots, I_{t}\right]$ es conocida. Utilizar la secuencia correcta es crítico, ya que la identificación de $\theta$ se basa en el cambio de un régimen cooperativo a uno competitivo (Agostini, 2006, p. 31). De acuerdo a Tilton et al. (1997), Agostini (2006) y la creencia general en el sector, la industria del cobre ha sido competitiva al menos desde 1980. Esta información permite fijar la secuencia $\left[I_{\text {ene1994 }}, \ldots, I_{d i c 2000}\right]$ igual a cero. Al mismo tiempo, con base en: las adquisiciones de Cyprus Amax Corp. y Asarco Inc. a finales de 1999 por parte de Phelps Dodge Corp. y Grupo México respectivamente; la fusión entre Freeport-McMoran C\&G y Phelps Dodge Corp en

7 Ver Boyd et al. (2003) y Jung et al. (1995). 
TABLA 5.1

TEST DE HIPOTESIS DE PODER DE MERCADO BAJO DIFERENTES ESTRUCTURAS DE MERCADO Y EL INDICE DE LERNER ESTIMADO PARA EL MODELO BL

\begin{tabular}{|l|c|c|}
\hline & Modelo 1 (MC2E) & Modelo 2 (MC3E) \\
\hline Monopolio & $\mathrm{H}_{0}: \lambda=1 ; \mathrm{H}_{1}: \lambda \neq 1$ & 0,0000 \\
Hipótesis & $\mathrm{H}_{0}: \lambda=1 ; \mathrm{H}_{1}: \lambda \neq 1$ \\
P-Valor & 0,0000 \\
Conclusión & Rechazar $\mathrm{H}_{0}$ al $1 \%$ de significancia & Rechazar $\mathrm{H}_{0}$ al $1 \%$ de significancia \\
Cournot & $\mathrm{H}_{0}: \lambda=0,2 ; \mathrm{H}_{1}: \lambda \neq 0,2$ & \\
Hipótesis & 0,0000 & $\mathrm{H}_{0}: \lambda=0,2 ; \mathrm{H}_{1}: \lambda \neq 0,2$ \\
P-Valor & 0,0000 \\
Conclusión & Rechazar $\mathrm{H}_{0}$ al $1 \%$ de significancia & Rechazar $\mathrm{H}_{0}$ al $1 \%$ de significancia \\
Competencia perfecta & $\mathrm{H}_{0}: \lambda=0 ; \mathrm{H}_{1}: \lambda \neq 0$ & 0,0096 \\
Hipótesis & $\mathrm{H}_{0}: \lambda=0 ; \mathrm{H}_{1}: \lambda \neq 0$ \\
P-Valor & 0,0065 \\
Conclusión & Rechazar $\mathrm{H}_{0}$ al $1 \%$ de significancia & Rechazar $\mathrm{H}_{0}$ al $1 \%$ de significancia \\
Índice de Lerner & & \\
L & 0,0070 & 0,0071 \\
\hline
\end{tabular}

Notas: Contraste de hipótesis utilizando el Test de Wald.

TABLA 5.2

TEST DE HIPOTESIS DE PODER DE MERCADO BAJO DIFERENTES ESTRUCTURAS DE MERCADO Y EL INDICE DE LERNER ESTIMADO PARA EL MODELO AP

\begin{tabular}{|c|c|c|}
\hline & Modelo 1 (MC2E) & Modelo 2 (MC3E) \\
\hline Régimen cooperativo & & \\
\hline Hipótesis & $\mathrm{H}_{0}: \theta=0,3 ; \mathrm{H}_{0}: \theta \neq 0,3$ & $\mathrm{H}_{0}: \theta=0,3 ; \mathrm{H}_{0}: \theta \neq 0,3$ \\
\hline P-Valor & 0,0000 & 0,0000 \\
\hline Conclusión & Rechazar $\mathrm{H}_{0}$ al $1 \%$ de significancia & Rechazar $\mathrm{H}_{0}$ al $1 \%$ de significancia \\
\hline Cournot & & \\
\hline Hipótesis & $\mathrm{H}_{0}: \theta=0,2 ; \mathrm{H}_{0}: \theta \neq 0,2$ & $\mathrm{H}_{0}: \theta=0,2 ; \mathrm{H}_{0}: \theta \neq 0,2$ \\
\hline P-Valor & 0,0000 & 0,0000 \\
\hline Conclusión & Rechazar $\mathrm{H}_{0}$ al $1 \%$ de significancia & Rechazar $\mathrm{H}_{0}$ al $1 \%$ de significancia \\
\hline Competencia perfecta & & \\
\hline Hipótesis & $\mathrm{H}_{0}: \theta=0 ; \mathrm{H}_{0}: \theta \neq 0$ & $\mathrm{H}_{0}: \theta=0 ; \mathrm{H}_{0}: \theta \neq 0$ \\
\hline P-Valor & 0,0035 & 0,0022 \\
\hline Conclusión & Rechazar $\mathrm{H}_{0}$ al $1 \%$ de significancia & Rechazar $\mathrm{H}_{0}$ al $1 \%$ de significancia \\
\hline Índice de Lerner & & \\
\hline $\begin{array}{l}\mathrm{L} \\
\text { Conclusión }\end{array}$ & 0,1387 & 0,1365 \\
\hline Conclusión & Mercado competitivo & Mercado competitivo \\
\hline
\end{tabular}

Notas: Contraste de hipótesis utilizando el Test de Wald. 
2006; y de acuerdo a la información reportada por O'Ryan (2002) y por Mining Deals (PWC, varios años) sobre las fusiones y adquisiciones de la industria minera se fija la secuencia $\left[I_{\text {ene2001 }}, \ldots, I_{d i c 2007}\right]$ igual a uno ${ }^{8}$. Por lo tanto, la secuencia restante $\left[I_{\text {ene2008 }}, \ldots, I_{\text {dic } 2009}\right]$ es igual a cero. La construcción de la secuencia completa $\left[I_{\text {ene } 1994, \ldots,}\right.$ $\left.I_{d i c 2009}\right]$ permitirá probar si la ola de fusiones y de adquisiciones cambia el régimen de competencia de la industria.

El coeficiente de la variable dummy, $I_{t}$, es positivo y significativo con un valor estimado cercano a 0,15 . Asumiendo que el coeficiente de $I_{t}, \gamma_{6}$ en la ecuación (27), es igual a $-\log \left(1+\theta / \omega_{1}\right)$ para algún valor constante $\theta$; entonces el valor de $\theta$ (parámetro de conducta en el modelo AP) puede ser calculado con los coeficientes estimados de $\gamma_{6}$ y $\omega_{1}(\mathrm{X})$, cuyo resultado como se muestra en la Tabla 4 presenta un valor de 0,0527 y 0,0532 para la estimación uno (MC2E) y dos (MC3E), respectivamente. Estos resultados sugieren que los precios son cercanos a los que predice la teoría bajo un régimen competitivo.

De forma similar que en el análisis del modelo BL, se realizan cuatro pruebas adicionales para confirmar el valor estimado del parámetro de conducta, $\theta$. Primero, se comprueba la conducta del mercado bajo un régimen cooperativo fijando $\theta=0,3$. Segundo, se fija una conducta a la Cournot con cinco firmas, $\theta=0,2$. Tercero, se prueba la hipótesis de competencia perfecta fijando $\theta=0$. Y por último, se construye el índice de Lerner como en (28). Los resultados de las distintas pruebas de conducta para el modelo AP se presentan en la Tabla 5.2.

Los resultados de los test de conducta confirman que la industria del cobre estuvo bajo un régimen competitivo durante el período 1994 y 2009.

De acuerdo a los resultados empíricos, a pesar de que el modelo AP presentó un parámetro de conducta superior al modelo BL, ambos modelos concluyen que las empresas no han ejercido poder de mercado durante el período analizado. Precisando que estos resultados deben interpretarse como un promedio total de la industria en el período de análisis. Lo que finalmente muestran los resultados es que los precios del cobre fueron en promedio muy cercanos a los precios competitivos durante el período que va desde 1994 hasta 2009.

Sin embargo, considerando el análisis anterior quedan algunas interrogantes, como por ejemplo ¿Cuál ha sido la causa de la ola de fusiones y adquisiciones de empresas en el sector minero? y ¿Cuál ha sido su impacto sobre la conducta de la industria? Considerando los resultados del modelo y el análisis de la industria surgen dos posibles explicaciones para la primera interrogante.

La primera es que las empresas han debido hacer frente a la creciente competencia externa a través de fusiones y adquisiciones. Las importaciones de cobre representaron en

8 El volumen de fusiones y adquisiciones en el sector minero se incrementa significativamente a partir del año 2001, manteniendo un alto volumen de transacciones hasta el año 2007. Durante el año 2008 el volumen transado en el sector de metales base en Norteamérica disminuye casi un $90 \%$ respecto del año anterior (PWC, 2008). 
promedio un 25\% del consumo local para el período de 1998 a 2002. Las importaciones podrían presionar el precio a la baja, por lo que la estimación del poder de mercado podría estar sesgado positivamente al no incluir explícitamente las importaciones en el modelo. Sin embargo, al estar el precio determinado en un mercado internacional y que la mayoría de los productores mundiales tienen operaciones en Estados Unidos, este efecto estaría internalizado en los precios, por lo que la magnitud del sesgo en la estimación del poder de mercado debería ser mínima9 .

Segundo, considerando las características del mercado mundial del cobre, en la que rige un precio internacional, las empresas locales han debido ajustarse y transformarse en empresas eficientes para poder sobrevivir, lo que ha tenido como consecuencia la modificación de la estructura de la industria y las ha obligado a pasar de ser una industria competitiva a nivel local a ser una competitiva a nivel global. Esta explicación puede haber justificado que el Congreso Norteamericano y la Comisión Federal de Comercio de Estados Unidos no hayan rechazado ninguna de estas fusiones y adquisiciones en la industria minera. A nivel general Susanto (2006) señala que una industria más eficiente conducirá a una mayor concentración. En la misma línea Demsetz (1973) plantea que algunas empresas son más eficientes que otras al producir el mismo bien a menor costo, las empresas más eficientes crecerán con el tiempo resultando en mayores y más eficientes empresas, lo que tendrá como consecuencia una industria más concentrada (por la mayor expansión de mercado de las empresas).

En cuanto a la segunda interrogante, considerando la explicación anterior y los resultados de la investigación, se podría considerar que la estructura competitiva de la industria no ha sido modificada.

\section{CONCLUSIONES}

El mercado del cobre en Estados Unidos ha estado altamente concentrado, las cinco mayores empresas aglutinaron más del 76\% de la producción en el año 2008. El índice de Herfindahl-Hirschman en ese año fue superior a los 1.800 puntos (3.000), el límite fijado por la legislación norteamericana antimonopolio y de la libre competencia a partir del cual el poder de mercado podría ser preocupante. Sin embargo, considerar solo este índice en el análisis de la industria puede conducir a conclusiones erróneas acerca del poder de mercado de la industria.

Debido a las condiciones del mercado mundial del cobre y los bajos precios de los minerales a partir de mediados de la década de los noventa, hasta la primera mitad del 2000, las empresas no han tenido otras herramientas para sortear estas dificultades más que por medio de recortes de producción y por fusiones y adquisiciones. Por lo

9 El autor agradece al referee por hacer notar el rol de las importaciones en la estimación del poder de mercado. Para un completo análisis sobre el efecto de las importaciones en la determinación de los precios de un mercado local el lector es referido a Salvo (2010). 
tanto, el principal objetivo de esta investigación fue estimar el poder de mercado en la industria del cobre de Estados Unidos utilizando modelos estructurales propuestos por la NOIE para el período entre 1994 y 2009.

Los resultados estadísticos sugieren que la demanda es sensible al precio y confirman una pendiente negativa. La elasticidad precio de la demanda estuvo entre los rangos estimados previamente en la literatura. Se concluye que los modelos de la NOIE estimados presentan un parámetro de conducta pequeño y significativamente diferente de cero, lo que es consistente con precios cercanos al nivel de precios de competencia perfecta. Esto puede ser interpretado como que la industria del cobre ha sido competitiva durante el período examinado. No obstante, este resultado debe ser interpretado solo como un promedio de la conducta de la industria durante este período, y es posible que la industria mantuviera alguna conducta colusiva ya sea en precios o cantidades, en intervalos cortos de tiempo durante este período.

Por lo tanto, a pesar de la alta concentración de la industria los resultados muestran que la ola de fusiones y adquisiciones pudiera no haber tenido efecto sobre el poder de mercado en el período analizado. Algunas posibles explicaciones se deben al intento de las empresas de soportar la competencia externa, mantener estrategias de control sobre los precios que eviten la entrada de nuevos competidores y por último buscar la eficiencia en costos, lo que necesariamente conducirá a una mayor concentración de la industria.

La metodología de la NOIE ha sido ampliamente utilizada. Sin embargo, no está exenta de presentar algunos problemas cuando se valida empíricamente. Por una parte, en el modelo BL es posible que una estimación lineal presente problemas de multicolinealidad (Perloff y Shen, 2001). En tanto que en el modelo AP los resultados pueden ser debatibles de acuerdo al criterio de construcción de la secuencia del régimen cooperativo, por ejemplo al considerar el efecto o no de fusiones y adquisiciones de la industria fuera del mercado norteamericano o el posible rezago de su efecto sobre el poder de mercado. No obstante estos potenciales problemas, los resultados obtenidos en esta investigación están ampliamente respaldados por los estudios previos.

Esta investigación constituye una primera aproximación a un estudio en profundidad de la industria del cobre. En virtud del estudio realizado y los resultados obtenidos es posible señalar que esfuerzos futuros de investigación debiesen considerar la mejora de la calidad de información disponible, como por ejemplo de las fuentes privadas que son habitualmente utilizadas en el análisis de la industria, lo que permitiría tener una base de datos más exacta, diversificada, amplia y completa con todos los factores que afecten a la industria. Por otra parte, es posible explorar modelaciones econométricas dinámicas, como por ejemplo una estimación por el mecanismo de corrección de error (MCE), que capture los posibles juegos colusivos de las empresas en períodos intermedios así como los patrones de conducta de corto y largo plazo. Finalmente, este estudio puede ser ampliado a un análisis del mercado mundial del cobre, lo que permitiría identificar las posibles estrategias de producción y control de precios de las empresas, que determinen el poder de mercado de la oferta en un contexto global. 


\section{REFERENCIAS BIBLIOGRAFICAS}

AGOSTINI, C. (2006). "Estimating Market Power in the US Copper Industry", Review of Industrial Organization, Vol. (28), pp. 17-39.

ALVARADO, F. y T. OVERBYE (1998). "Measuring Reactive Market Power", Report PSERC, 29.

APPELBAUM, E. (1982). "The Estimation of the Degree of Oligopoly Power", Journal of Econometrics, Vol. (19), pp. 287-299.

BHP BILLITON (varios años). "Annual Report”, BHP.

BOYD, R.; C. JUNG y B. SELDON (1995). "The Market Structure of the US Aluminum Industry”, Journal of Economics and Business, Vol. (47), pp. 293-301.

BRESNAHAN, T. (1982). "The Oligopoly Solution Concept is Identified”, Economics Letters, Vol. (10), pp. 87-92.

BRESNAHAN, T. (1987). "Competition and Collusion in the American Automobile Industry: The 1955 Price War", The Journal of Industrial Economics, Vol. (35), pp. 457-482.

BRESNAHAN, T. (1989). "Empirical Studies of Industries with Market Power" en Richard Schmalensee y Robert D. Willing, Handbook of Industrial Organization, Vol II: chapter 17, Elsevier Science Publishers, pp. 1011-1057.

CDA (2010). "Annual data 2010: copper-brass-bronze, copper supply \& consumption 1988-2009", Nueva York.

COCHILCO (2008). “Informe del mercado del cobre. Análisis 2008 y perspectivas 2009-2010”, Comisión Chilena del Cobre.

CODELCO (varios años). "Memoria Annual”, Codelco-Chile.

DEMSETZ, H. (1973). "Industry Structure, Market Rivalry, and Public Policy,” Journal of Law \& Economics, Vol. (16), pp. 1-9.

ENGLE, R. y C. GRANGER (1987). "Co-Integration and Error Correction: Representation, Estimation, and Testing". Econometrica, Vol. (55), pp. 251-276.

FOLEY, P. y J. CLARK (1981). "US Copper Supply an Economic/Engineering Analysis of Cost-Supply Relationships", Resources Policy, Vol. (7), pp. 171-187.

GALLET, C. (2001). "The Gradual Response of Market Power to Mergers in the U.S. Steel Industry", Review of Industrial Organization, Vol. (18), pp. 327-336.

GARCIA, P. y R. M. LEUTHOLD (1997). "Commodity Market Modeling" en Padberg, D.I.; C. Ritson, y L.M. Albisu, Agro-food Marketing, CAB International, Oxford, pp. 111-134.

GENESOVE, D. y W. MULLIN (1998). "Testing Static Oligopoly Models: Conduct and Cost in the Sugar Industry, 1890-1914", The RAND Journal of Economics, Vol. (29), pp. 355-377.

GRANGER, C. y P. NEWBOLD (1974). "Spurious Regressions in Econometrics", Journal of Econometrics, Vol. (2), pp. 111-120.

GREENE, W. (2003). Econometric Analysis. 5th Edición, Prentice Hall.

GUZMAN, J. I. (2007). Essays in Industrial Organization Applied to Mineral Markets. Thesis of the requirements for the Degree of Doctor in Engineering Sciences, Pontificia Universidad Católica de Chile, Escuela de Ingeniería.

HENDRY, D. (1991). "Econometric Modeling with Cointegrated Variables: An Overview" en R. F. Engle y C.W.J. Granger, Long-run Economic Relationships: Readings in Cointegration. Oxford University Press, pp. 51-64.

ICSG (1994-2010). Monthly Copper Usage Data: Monthly Copper Mine Production Data. International Copper Study Group.

IGAMI, M. (2015). "Market Power in International Commodity Trade", The Journal of Industrial Economics, Vol. (63), pp. 225-248

JUNG, C. y B. SELDON (1995). "The Degree of Competition in the Advertising Industry", Review of Industrial Organization, Vol. (10), pp. 41-52.

LAU, L. (1982). "On Identifying the Degree of Competitiveness from Industry Price and Output Data", Economics Letters, Vol. (10), pp. 93-99. 
O'RYAN, R. (2002). "Fusiones y poder de mercado en la industria mundial del cobre". Centro de Economía Aplicada, Departamento de Ingeniería Industrial, Facultad de Ciencias Físicas y Matemáticas, Universidad de Chile.

PERLOFF, J. y E. SHEN (2001). "Collinearity in Linear Structural Models of Market Power", Working Paper Series from Institute of Industrial Relations, No 1020, UC Berkeley.

PORTER, R. (1983). "A Study of Cartel Stability: The Joint Executive Committee, 1880-1886”, The Bell Journal of Economics, Vol. (14), pp. 301-314.

PRICE WATER HOUSE COOPERS (varios años). "Mining Deals: Annual Review".

SALVO, A. (2010). "Inferring Market Power under the Threat of Entry: The Case of the Brazilian Cement Industry", The RAND Journal of Economics, Vol. (41), pp. 326-50.

SUSANTO, D. (2006). Measuring The Degree of Market Power in the Export Demand for Soybean Complex. Submitted to the Graduate Faculty of the Louisiana State University and Agricultural and Mechanical College in partial fulfillment of the requirements for the degree of Doctor of Philosophy.

TILTON, J. y H. LANDSBERG (1997). "Innovation, Productivity Growth, and the Survival of the U.S. Copper Industry". Discussion Paper, 97/41, series de estudios "Understanding Productivity Change in Natural Resource Industries".

U.S. BUREAU OF LABOR STATISTICS. Databases \& Tables (en línea). "www.bls.gov". (consulta: Abril de 2010)

U.S. ENERGY INFORMATION ADMINISTRATION. Energy Statistics (en línea). "www.eia.doe.gov". (consulta: Abril de 2010).

U.S. GEOLOGICAL SURVEY (1994-2010) Monthly prices Data: Copper, aluminum and silver. U.S. Geological Survey. National Center, Reston, VA.

U.S. GEOLOGICAL SURVEY (1994-2008) “U.S. Geological Survey Minerals Yearbook: Copper”. U.S. Geological Survey. National Center, Reston, VA.

U.S. SECURITIES AND EXCHANGE COMMISSION (SEC). Form 10-k. "http://www.sec.gov" (consulta: Julio de 2010).

U.S. FEDERAL RESERVE. ECONOMIC. Research \& Data (en línea). "http://www.federalreserve.gov" (consulta: Julio de 2010).

WOLFRAM, C. (1999). "Measuring Duopoly Power in the British Electricity Spot Market", The American Economic Review, Vol. (89), pp. 805-826.

ZEIDAN, R. y M. RESENDE (2009). "Measuring Market Conduct in the Brazilian Cement Industry: A Dynamic Econometric Investigation”, Review of Industrial Organization, Vol. (34), pp. 231-244. 


\section{ANEXOS}

\section{Anexo 1: Pruebas de raíz unitaria y cointegración}

Es ampliamente reconocido que varias series de interés económico, utilizadas en el análisis de series de tiempo, son no estacionarias, por lo que su uso para estimar relaciones de largo plazo puede conducir a relaciones espurias (Engle y Granger, 1987). Algunas perspectivas sugieren que para solucionar este problema las variables pueden ser transformadas a primeras diferencias; no obstante, es posible que información económica relevante de largo plazo se pierda si los datos son solo analizados en primeras diferencias $\left(\right.$ Hendry, 1986) ${ }^{10}$. Afortunadamente, la abundante literatura respecto al tema muestra otras alternativas para tratar el problema. Específicamente, siguiendo a Greene (2003), al mantener un grupo de variables integradas de orden uno (no estacionarias) que están cointegradas, en un modelo de regresión que esté lo suficientemente bien especificado, sus residuos serán una serie estacionaria que evitarán el problema de regresión espuria ${ }^{11}$.

Siguiendo esta propuesta, para verificar que el modelo econométrico a ser estimado cumple con las condiciones de estacionariedad se siguen dos líneas de exploración. En primer lugar, se verificó el orden de integración de las variables bajo consideración. Para esto se utilizaron las pruebas de raíz unitaria Dickey-Fuller Aumentado (ADF), Phillips-Perron (PP) y Kwiatkowski (KPSS) ${ }^{12}$. Los resultados de las dos primeras pruebas ADF y PP, que se muestran en la Tabla A.1, indican que todas las variables siguen un proceso integrado de orden uno, I(1). Adicionalmente, para corroborar los anteriores resultados se realizó la prueba KPSS (incluyendo constante e incluyendo constante y tendencia), para un rango de rezago desde cero a cuatro. En general los resultados que se muestran en la Tabla A.2 indican que la hipótesis nula de estacionariedad se rechaza a un nivel de significancia de 5\%. Dado que la hipótesis nula de la prueba KPSS es que la serie es estacionaria, su rechazo confirma los resultados obtenidos en las pruebas ADF y PP de raíz unitaria de la Tabla A.1.

10 Esta afirmación es válida solo si el proceso generado por las variables están cointegradas. En caso contrario, una buena solución será inferir un análisis en primeras diferencias (Hamilton, 1994).

11 Granger y Newbold (1974) denominan problema de regresión espuria al momento en que se analizan múltiples variables independientes, pudiendo ser todas $\mathrm{I}(1)$ o algunas $\mathrm{I}(0)$, y los estadísticos usuales indican una relación significativa cuando en realidad no la hay.

12 Las pruebas de raíz unitaria ADF y PP contrastan la hipótesis nula de raíz unitaria contra la hipótesis alternativa de no raíz unitaria. La prueba KPSS contrasta la hipótesis nula de estacionariedad contra la alternativa de no estacionariedad. 
TABLA A.1

PRUEBA DE RAIZ UNITARIA ADF Y PP

\begin{tabular}{|l|l|r|r|r|r|r|r|}
\hline \multirow{2}{*}{ Variable } & & \multicolumn{3}{|c|}{ ADF } & \multicolumn{3}{c|}{ PP } \\
\cline { 3 - 7 } & & \multicolumn{1}{|c|}{$\mathrm{A}$} & \multicolumn{1}{c|}{$\mathrm{B}$} & $\mathrm{C}$ & $\mathrm{A}$ & $\mathrm{B}$ & \multicolumn{1}{c|}{$\mathrm{C}$} \\
\hline Precio del cobre & $\mathrm{P}$ & $-1,3809$ & $-2,1564$ & 0,1087 & $-1,3025$ & $-2,0448$ & 0,1844 \\
Îndice de producción & & & & & & & \\
de la construcción & $\mathrm{CPI}$ & $-2,4621$ & $-2,2763$ & $-0,8385$ & $-2,4898$ & $-2,4945$ & $-0,2616$ \\
Precio del aluminio & $\mathrm{Z}$ & $\mathbf{- 3 , 2 2 6 5}$ & $-3,3098$ & $-0,1268$ & $\mathbf{- 3 , 3 4 1 4}$ & $\mathbf{- 3 , 4 4 0 0}$ & $-0,1986$ \\
Consumo de cobre & $\mathrm{Q}$ & 0,3767 & $-1,7205$ & $-1,3019$ & $\mathbf{- 3 , 5 6 3 1}$ & $\mathbf{- 5 , 1 7 4 6}$ & $-1,1213$ \\
Salario & $\mathrm{W}$ & $-1,7319$ & $-2,0071$ & $-0,4815$ & $-1,2630$ & $-1,4889$ & $-0,5008$ \\
Precio del petróleo & $\mathrm{OIL}$ & $-1,8786$ & $\mathbf{- 4 , 3 5 4 2}$ & $-0,1343$ & $-1,7745$ & $\mathbf{- 3 , 6 7 2 8}$ & $-0,0676$ \\
Precio de la electricidad & $\mathrm{E}$ & $-1,8677$ & $-1,9754$ & $-0,2515$ & $-2,8814$ & $\mathbf{- 2 , 9 3 0 1}$ & $-0,5573$ \\
Precio de la plata & SIL & $-0,3098$ & $-2,4619$ & 0,8639 & $-0,5687$ & $-2,1500$ & 0,7493 \\
\hline
\end{tabular}

Notas: Los valores en negrita indican el rechazo de la hipótesis nula al 5\% de significancia. El valor crítico del modelo que incluye constante (A) es de $-2,8775$, para el modelo que incluye constante y tendencia (B) es de $-3,4336$ y para el modelo que no incluye ni constante ni tendencia (C) es de $-1,9425$. Cálculos propios utilizando el paquete econométrico EViews 5.

TABLA A. 2

PRUEBA DE RAIZ UNITARIA KPSS

\begin{tabular}{|c|c|c|c|c|c|c|}
\hline \multirow{2}{*}{ Variable } & & \multicolumn{5}{|c|}{ Nivel } \\
\hline & & 0 & 1 & 2 & 3 & 4 \\
\hline Precio del cobre & $\mathrm{P}$ & 8,9085 & 4,5217 & 3,0573 & 2,3251 & 1,8866 \\
\hline $\begin{array}{l}\text { Índice de producción } \\
\text { de la construcción }\end{array}$ & CPI & 4,3154 & 2,2361 & 1,5470 & 1,2087 & 1,0096 \\
\hline Precio del aluminio & $\mathrm{Z}$ & 0,8147 & 0,4235 & 0,2927 & 0,2277 & 0,1892 \\
\hline Consumo de cobre & Q & 11,2647 & 6,1297 & 4,2590 & 3,2893 & 2,6915 \\
\hline Salario & W & 5,2782 & 2,6507 & 1,7756 & 1,3394 & 1,0785 \\
\hline Precio del petróleo & OIL & 14,6119 & 7,4181 & 5,0206 & 3,8264 & 3,1125 \\
\hline Precio de la electricidad & E & 2,4624 & 1,2665 & 0,8740 & 0,6811 & 0,5665 \\
\hline Precio de la plata & SIL & 10,4926 & 5,4019 & 3,6971 & 2,8416 & 2,3276 \\
\hline \multirow{2}{*}{ Variable } & & \multicolumn{5}{|c|}{ Tendencia } \\
\hline & & 0 & 1 & 2 & 3 & 4 \\
\hline Precio del cobre & $\mathrm{P}$ & 2,8766 & 1,4626 & 0,9917 & 0,7568 & 0,6164 \\
\hline $\begin{array}{l}\text { Índice de producción } \\
\text { de la construcción }\end{array}$ & CPI & 1,9034 & 0,9924 & 0,6907 & 0,5434 & 0,4574 \\
\hline Precio del aluminio & $\mathrm{Z}$ & 0,5816 & 0,3030 & 0,2097 & 0,1634 & 0,1359 \\
\hline Consumo de cobre & Q & 1,8460 & 1,0942 & 0,7945 & 0,6327 & 0,5296 \\
\hline Salario & W & 3,7708 & 1,8933 & 1,2683 & 0,9569 & 0,7709 \\
\hline Precio del petróleo & OIL & 2,8766 & 1,4626 & 0,9917 & 0,7568 & 0,6164 \\
\hline Precio de la electricidad & E & 1,4953 & 0,7693 & 0,5314 & 0,4147 & 0,3455 \\
\hline Precio de la plata & SIL & 2,9148 & 1,5223 & 1,0571 & 0,8241 & 0,6848 \\
\hline
\end{tabular}

Notas: Los valores en negrita indican el rechazo de la hipótesis nula al 5\% de significancia. El valor crítico al $5 \%$ en el modelo en nivel es de 0,463 y para el modelo con tendencia es de 0,146. Cálculos propios utilizando el paquete econométrico EViews 5. 
En segundo lugar, para verificar la existencia de relaciones de largo plazo entre las variables se realizó la prueba de cointegración de Johansen ${ }^{13}$ para cada una de las ecuaciones estimadas. Los resultados que se presentan en la Tabla A.3 comprueban la existencia de cointegración de las variables en cada una de las ecuaciones. Como se observa en los resultados, la hipótesis nula de no cointegración, $r=0$, se rechaza a un nivel del 5\%, tanto para el estadístico de traza como para el estadístico de máximo, lo que sugiere que existe al menos una relación de cointegración.

\section{TABLA A. 3}

TEST DE COINTEGRACION DE LAS VARIABLES EN LA ECUACION DE DEMANDA Y EN LAS RELACIONES DE OFERTA

\begin{tabular}{|l|c|c|c|}
\hline \multicolumn{1}{|c|}{ Variable } & \multicolumn{3}{c|}{ Hipótesis } \\
\cline { 2 - 4 } & $\mathrm{r}=0$ & $\mathrm{r} \leq 1$ & $\mathrm{r} \leq 2$ \\
\hline Ecuación de demanda & & & \\
Estadístico de traza & $\mathbf{5 4 , 3 9 7 4}$ & 24,0796 & 8,6357 \\
Valor crítico & 47,8561 & 29,7971 & 15,4947 \\
Estadístico de máximo & $\mathbf{3 0 , 3 1 7 8}$ & 15,4439 & 8,6075 \\
Valor crítico & 27,5843 & 21,1316 & 14,2646 \\
Relación de oferta Bresnahan \& Lau (1982) & & & \\
Estadístico de traza & $\mathbf{1 3 8 , 2 1 2 3}$ & $\mathbf{8 2 , 9 9 6 2}$ & 47,0434 \\
Valor crítico & 95,7537 & 69,8189 & 47,8561 \\
Estadístico de máximo & $\mathbf{5 5 , 2 1 6 1}$ & $\mathbf{3 5 , 9 5 2 8}$ & 21,9293 \\
Valor crítico & 40,0776 & 33,8769 & 27,5843 \\
Relación de oferta Porter (1983) \& Agostini (2006) & & & \\
Estadístico de traza & $\mathbf{1 0 2 , 7 8 4 9}$ & $\mathbf{5 0 , 2 4 3 1}$ & 27,5175 \\
Valor crítico & 69,8189 & 47,8561 & 29,7971 \\
Estadístico de máximo & $\mathbf{5 2 , 5 4 1 9}$ & $\mathbf{2 2 , 7 2 5 6}$ & 20,7707 \\
Valor crítico & 33,8769 & 27,5843 & 21,1316 \\
\hline
\end{tabular}

Notas: Los valores en negrita indican el rechazo de la hipótesis nula al 5\% de significancia. Los valores críticos se presentan en la taba. Cálculos propios utilizando el paquete econométrico EViews 5.

13 Engle y Granger (1987) señalan que una combinación lineal de dos o más series no estacionarias puede ser estacionaria. Si tal combinación lineal existe, las series no estacionarias están cointegradas y puede ser interpretada como una relación de equilibrio de largo plazo entre las series. Por lo tanto, el propósito del test de cointegración de Johansen es determinar si un grupo de series no estacionarias están cointegradas. 